\title{
Resistência à traça-do-tomateiro em plantas com altos teores de acilaçú- cares nas folhas
}

\author{
Álvaro C Gonçalves Neto' ${ }^{1}$ Vanisse de F Silva ${ }^{1}$; Wilson Roberto Maluf'; Gabriel M Maciel ${ }^{1}$; Daniela AC \\ Nízión $^{2}$; Luiz AA Gomes ${ }^{1}$; Sebastião M de Azevedo ${ }^{3}$ \\ ${ }^{1}$ UFLA-Depto. Agricultura, C. Postal 3037, 37200-000 Lavras-MG; ${ }^{2}$ UFLA-Depto. Biologia; ${ }^{3}$ Sakata Sudamerica, Bragança Paulista-SP; \\ alvarocgneto@gmail.com; vanissesilva@yahoo.com; wrmaluf@ufla.br; gmmufla@gmail.com; danielanizio@hotmail.com; laagomes@ \\ ufla.br; sebastiao.azevedo@sakata.com.br
}

\section{RESUMO}

A traça-do-tomateiro foi constatada pela primeira vez no Brasil em 1980 estando hoje dentre as principais pragas da tomaticultura nacional. O controle desta praga é feito principalmente por inseticidas, sendo realizada em casos extremos, até três pulverizações semanais. Acessos silvestres de tomateiro como $S$. pennellii LA-716 possuem resistência a pragas, mediada pela presença de acilaçúcares nos folíolos. Os objetivos deste trabalho foram obter genótipos de tomateiro com características comerciais e altos teores foliares de acilaçúcares (AA), e avaliar seus níveis de resistência à traça do tomateiro Tuta absoluta. Os genótipos foram obtidos a partir de populações segregantes $\left(\mathrm{F}_{2}\right)$ oriundas do cruzamento entre AF-8665 (linhagem elite com baixos teores de AA) e as linhagens BPX370E-30-27511-7, BPX370E-30-275-11-8, BPX370E-30-380-68-6 e BPX370E30-380-68-8 (linhagens pré-comerciais com altos teores, obtidas a partir do cruzamento interespecífico $S$. lycopersicum x S. pennellii LA-716). Plantas $\mathrm{F}_{2}$ previamente selecionadas com base nos teores de AA foram clonadas e em seguida testadas quanto à resistência à traça-do-tomateiro. Genótipos selecionados para altos teores de AA expressaram menor ovoposição da traça e níveis inferiores de danos causados à planta pela infestação do microlepidóptero do que genótipos (comerciais ou não) com baixos teores de AA. Embora genótipos com altos teores de AA tenham sido mais resistentes à traça do que os demais, seus níveis de resistência podem ser dependentes do background genotípico: clones BPX-410H (alto teor de AA) mostraram-se em geral ligeiramente mais resistentes do que o dos clones BPX-370G (também alto teor de AA). Os clones BPX-410H-01pl\#281, BPX-410H-04pl\#348 e BPX-410H-04pl\#481 foram superiores nas avaliações de resistência à traça e são recomendados para a continuidade do programa de melhoramento.

Palavras-chave: Solanum pennellii, Solanum lycopersicum, Tuta absoluta, aleloquímico.

\begin{abstract}
Resistance to the South American tomato pinworm in tomato plants with high foliar acylsugar contents
\end{abstract}

The South American tomato pinworm was first detected in Brazil in 1980 and today it is among the major pests of tomato in Brazil. The control of this pest has been done primarily with the use of insecticides, and three sprays per week may be necessary in extreme cases. Wild tomato accessions such as $S$. pennellii LA-716 possess high levels of insect resistance, mediated by high foliar contents of acylsugars. The objectives of this study were obtain improved tomato genotypes with high contents of leaf acylsugars, and to assess their levels of resistance to the South American tomato pinworm Tuta absoluta. These genotypes were obtained from segregating $\left(\mathrm{F}_{2}\right)$ populations from the cross between AF-8665 (an elite low AS tomato line) and lines BPX370E-30-275-11-7, BPX370E-30-275-11-8, BPX370E-30-380-68-6 and BPX370E-30-380-68-8 (pre-commercial high AS inbreds derived from an original interspecific cross between S. lycopersicum $\mathrm{x}$ S. pennellii LA-716). $\mathrm{F}_{2}$ plants selected for high AS contents were cloned and subsequently tested for pinworm resistance. The high AS genotypes showed smaller ovoposition counts and lower plant damage levels than low AS genotypes (commercial or not). Even though high AS genotypes were generally more resistant to the South American tomato pinworm than low AS genotypes, their actual levels of resistance may be background-dependent: high AS clones with BPX-410H background had slightly higher levels of resistance than high AS clones with BPX-370G background. Clones BPX-410H01pl\#281, BPX-410H-04pl\#348 and BPX-410H-04pl\#481 had the highest levels of pinworm resistance, and were recommended for further use in breeding programs.

Keywords: Solanum pennellii, Solanum lycopersicum, Tuta absoluta, allelochemical.

\section{(Recebido para publicação em 13 de julho de 2009; aceito em 10 de maio de 2010)} (Received on July 13, 2009; accepted on May 10, 2010)

\begin{abstract}
$\mathrm{A}$ traça-do-tomateiro (Tuta absoluta Meyr.) é atualmente uma das pragas mais importantes do tomateiro cultivado no Brasil. Espécies silvestres de tomateiro têm sido amplamente utilizadas como fonte de resistência a pragas e doenças no melhoramento de cultivares comerciais, particularmente a espécie Solanum pennellii. Os acessos de $S$. pennellii possuem altos níveis de resistência a um grande número de
\end{abstract}

artrópodos-praga, inclusive à traça-dotomateiro (Resende, 2003; Resende et al., 2006; Resende et al., 2008; Pereira et al., 2008). A resistência obtida a partir de $S$. pennellii tem sido associada à presença de aleloquímicos denominados de acilaçúcares (AA), que são ésteres de ácidos graxos. Estes aleloquímicos podem atuar impedindo a ovoposição, a alimentação ou, ainda, exercendo efeito deletério no desenvolvimento de determinadas fases de um artrópodopraga. Ao contrário do S. pennellii, as cultivares de tomates comerciais atuais não apresentam níveis elevados de AA, enquanto plantas $\mathrm{F}_{1}$ do cruzamento entre S. lycopersicum x $S$. pennellii podem apresentar níveis moderados (Resende et al., 2002).

Resende (2003) trabalhou com plantas selecionadas para alto e baixo teor de AA na população $\mathrm{F}_{2}$ do cruzamento $S$. 
lycopersicum ('TOM-584') x S. pennelli 'LA-716' e na população $\mathrm{F}_{2}$ do primeiro retrocruzamento para $S$. lycopersicum. Estas plantas foram submetidas a ensaios de repelência ao ácaro Tetranychus evansi, de resistência à mosca-branca (Bemisia sp.) e à traça-do-tomateiro ( $T$. absoluta Meyr.), juntamente com os genitores. Os resultados obtidos demonstraram o efeito dos AA na repelência ao ácaro. Com relação à mosca-branca, verificou-se menor número de ninfas e, com relação à traça, menores níveis de danos às plantas em genótipos com elevado teor de AA.

A obtenção de plantas com alto teor de AA nos folíolos e bons níveis de resistência às principais pragas do tomateiro é de grande interesse no manejo da cultura, na qual o controle de pragas tem sido realizado, basicamente, por meio de controle químico associado a outras práticas. Gonçalves et al. (2007) indicaram que altos teores de AA se devem à ação de um alelo recessivo, com dominância incompleta no sentido de altos teores. Em virtude da dominância ser incompleta, obter linhagens avançadas com altos teores foliares de AA poderia tornar possível a obtenção de híbridos resistentes a artrópodos-pragas mesmo entre linhagens com altos teores de AA e linhagens com baixos teores de AA, o que foi comprovado por Maluf et al. (2010). Este autor testando diferentes combinações híbridas entre linhagens com altos teores e linhagens com baixos teores de AA comprovou moderado nível de resistência ao ácaro (Tetranychus urticae) e níveis satisfatórios de resistência tanto para mosca branca (Bemisia argentifolii) quanto para a traça do tomateiro (T. absoluta Meyr.).

Embora a resistência a pragas em tomateiro mediada por AA derivados de $S$. pennellii seja bem documentada (Resende, 2003; Gonçalves et al., 2006; Resende et al., 2006; Saeidi et al., 2007; Maluf et al. (2010)), ainda não estão disponíveis no mercado tomateiros comerciais com níveis satisfatórios de resistência. Este trabalho teve por objetivos obter genótipos de tomateiro com altos teores foliares de AA, em diferentes backgrounds genotípicos com características comerciais e avaliar seus níveis de resistência à traça
(Tuta absoluta Meyr.) relativamente às testemunhas comerciais, com baixos teores de AA.

\section{MATERIAL E MÉTODOS}

\section{Obtenção e seleção de genótipos de tomateiro com altos teores de $\mathrm{AA}$}

- Foi realizado um ensaio experimental no período de novembro de 2005 a fevereiro de 2006, em casa de vegetação aberta lateralmente na Estação Experimental de Hortaliças-HortiAgro Sementes Ltda, Fazenda Palmital, Ijaci-MG (21 ${ }^{\circ} 14^{\prime} 16^{\prime \prime d e ~ l a t i t u d e ~ s u l ~ e ~}$ 4500'00" de longitude, com $918 \mathrm{~m}$ de altitude). As avaliações foram realizadas no Laboratório de Química Orgânica, do Departamento de Química, da Universidade Federal de Lavras. Para a seleção de genótipos com altos teores de AA, utilizaram-se quatro populações segregantes $\left(\mathrm{F}_{2}\right)$ oriundas do cruzamento entre AF-8665 (linhagem elite de crescimento determinado, com baixo teor de $\mathrm{AA}$, pertencente à empresa Sakata Seed Corporation) e os genótipos BPX-370E30-275-11-7, BPX-370E-30-275-11-8, BPX-370E-30-380-68-6 e BPX-370E30-380-68-8 (genótipos pré-comerciais de crescimento indeterminado, com altos teores de $\mathrm{AA}$, pertencentes à HortiAgro Sementes Ltda., e acessos do $2^{\circ}$ retrocruzamento para $S$. lycopersicum 'TOM-584', a partir do cruzamento interespecífico original entre S. lycopersicum x S. pennellii LA-716). Estas populações $\mathrm{F}_{2}$ foram denominadas BPX410H-01, BPX-410H-02, BPX-410H03, e BPX-410H-04, respectivamente, e representam populações $\mathrm{F}_{2} \mathrm{RC}_{3}$ oriundas do terceiro retrocruzamento para $S$. lycopersicum, a partir do cruzamento original S. lycopersicum x S. pennellii 'LA-716'. Foram semeadas em bandejas de poliestireno expandido e posteriormente transplantadas para vasos de 500 $\mathrm{mL}$ totalizando 500 plantas, das quais 400 pertenciam às populações $\mathrm{F}_{2}$ (100 plantas de cada uma das quatro populações BPX-410H), 50 da testemunha para alto teor de AA (S. pennelli 'LA-716') e 50 da testemunha para baixo teor de AA (S. lycopersicum 'TOM-584'), uma linhagem de crescimento indeterminado de tipo comercial e background genotípico similar à cultivar Santa Clara. As plantas foram individualmente avaliadas quanto aos teores de AA nos folíolos, de acordo com metodologia para a determinação de açúcares redutores (Resende et al., 2002) em duas observações por planta, sendo o teor de AA estimado por meio da média das duas observações para cada planta. Dentre as quatro populações, as 30 plantas $\mathrm{F}_{2}$ que apresentaram teores mais elevados de AA foram preliminarmente selecionadas, e reavaliadas quanto ao teor de AA (média de seis observações repetidas para cada planta) seguindo a mesma metodologia. Foram selecionadas as quinze plantas que apresentaram teores mais elevados de AA, correspondentes a valores entre 65 e $97,4 \%$ do teor médio encontrado em folhas de S. pennellii LA-716 (em média, 75,3\%) (Tabela 1). Estas plantas foram clonadas via enraizamento de brotações axilares com tamanhos homogêneos. Em seguida, os seis genótipos com altos teores de AA que disponibilizaram número suficiente (no mínimo quatro) de plantas clonadas: clones BPX-410H-01pl\#281 (T9), BPX-410H-02pl\#69 (T10), BPX-410H-03pl\#345 (T11), BPX-410H-03pl\#368 (T12), BPX-410H-04pl\#348 (T13) e BPX410H-04pl\#481 (T14) foram incluídos no experimento para avaliação de resistência à traça do tomateiro (T. absoluta Meyr.). Foram também utilizados nos estudos outros clones disponíveis na HortiAgro Sementes Ltda., previamente selecionados para alto teor de AA: BPX370G-30-380-68-08-02pl\#192 (T1), BPX-370G-30-380-68-08-02pl\#287 (T2), BPX-370G-30-380-68-0804pl\#269 (T3), BPX-370G-30-38068-08-05pl\#270 (T4) e BPX-370G-30380-68-08-05pl\#283 (T5), assim como três clones selecionados com baixos teores de AA, BPX-370G-30-380-680802pl\#045 (T6), BPX-370G-30-38068-08-04pl\#036 (T7) e BPX-370G-30380-68-08-05pl\#120 (T8). Estes clones BPX-370G (tanto os de altos quanto os de baixos teores de AA) foram obtidos a partir de populações $\mathrm{F}_{2} \mathrm{RC}_{3}$ oriundas do terceiro retrocruzamento para $S$. lycopersicum a partir do cruzamento original $S$. lycopersicum x $S$. pennellii 'LA-716', em que o genitor recorrente S. lycopersicum 'TOM-584' possuía um background genotípico distinto da 
AF-8665. Os procedimentos adotados para a obtenção dos clones BPX-370G foram semelhantes aos empregados na obtenção dos clones BPX-410H. Todos os clones BPX-370G e BPX-410H selecionados possuíam características comparáveis a tomateiros comerciais, e eram portanto bastante distintos do acesso $S$. pennellii 'LA-716' usado originalmente no cruzamento interespecífico como fonte da característica alto teor de AA, e agronomicamente superiores a ele.

Reação de genótipos de tomateiro à infestação com a traça $T$. $\boldsymbol{a} \boldsymbol{b}$ soluta - Os genótipos selecionados em relação ao teor de AA foram avaliados quanto à resposta a infestação de traça-do-tomateiro (T. absoluta Meyr.) em experimento conduzido entre agosto de 2006 e novembro de 2006 . Os materiais genéticos utilizados neste experimento foram: (a) seis clones com altos teores de AA selecionados na primeira etapa deste trabalho (T9, T10, T11, T12, T13 e T14); (b) cinco clones com altos teores de AA com outro background genotípico (T1, T2, T3, T4 e T5); (c) três clones com baixos teores de AA (T6, T7 e T8). Como testemunhas foram utilizados o genótipo 'LA-716'(T17), as linhagens comerciais TOM-584 (T15) e TOM-598 (T16) e o híbrido comercial Bônus $F_{1}$ (baixo teor de AA) (T18).

As mudas dos genótipos-testemunha foram produzidas via sementes (sem a necessidade de utilização de estaquia). Para a infestação com T. absoluta, foi previamente estabelecida uma criação de traça-do-tomateiro em estufa telada, constituída por uma estrutura de proteção de 12 m² (4 x 3 m), modelo capela, com cobertura de plástico transparente de 100 micras de espessura e laterais de tela antiafídica. A infestação na estufa telada foi efetuada com plantas visivelmente atacadas da cultivar suscetível Santa Clara, às quais se acrescentavam novas plantas suscetíveis a cada 15 dias, de modo a manter continuamente altas populações de inóculo dentro da estufa telada; contudo, a quantidade efetiva de adultos no inóculo inicial não foi determinada. Em agosto de 2006 foi realizada a semeadura das testemunhas (linhagens e híbrido) em bandejas de poliestireno com 128 células utilizando substrato Plantmax $^{\circledR}$. Em outubro de 2006 foi feito o transplantio das mudas dos clones (anteriormente obtidos via enraizamento de brotações axilares) e das testemunhas para vasos de 3,44 L. Decorridos quinze dias após o transplantio os diferentes tratamentos foram colocados na estufa telada previamente infestada com uma população de T. absoluta. Aos 14 dias após infestação, foi realizada avaliação da ovoposição, por meio da contagem do número de ovos com o auxílio de um microscópico estereoscópico binocular com aumento de 20 a 80 vezes (número de ovos em $2 \mathrm{~cm}^{2}$ de área foliar), a avaliação foi repetida após quatro dias, tomando como número total de ovos a média das duas contagens. Foram utilizados para as contagens apenas folíolos do terço superior da planta, previamente marcados com uma fita adesiva branca para posteriores avaliações no mesmo local amostrado. O delineamento utilizado foi inteiramente casualizado com uma planta (vaso) por parcela e quatro repetições por tratamento. A temperatura média e a umidade relativa do ar entre o período de infestação das plantas até o último dia de avaliação dos danos variaram de 18,5 a $27,4^{\circ} \mathrm{C}$ e 84,5 a $100 \%$, respectivamente. Iniciando-se aos 20 dias após a infestação, as plantas foram individualmente avaliadas quanto aos níveis de danos de acordo com escalas de notas de um (menor dano) a cinco (maior dano), propostas por Maluf et al. (1997) e Labory et al. (1999) para os seguintes parâmetros: lesões no folíolo (LF) e porcentagem de folíolos atacados (PFA) e danos gerais à planta (DP).

Análise dos dados - Foram feitas nove avaliações, uma a cada três dias, a partir do $20^{\circ}$ dia após a infestação. As médias das notas (dados transformados $\sqrt{(x+1 / 2)})$ e o número total de ovos foram submetidos à análise de variância utilizando o teste de agrupamento de Scott-Knott a 5\% de probabilidade por meio do aplicativo estatístico SISVAR (Ferreira, 2000). Contrastes entre tratamentos de altos e baixos teores de AA foram estimados, de modo a obter melhores inferências sobre a relação entre teor de AA e os níveis de resistência à traça.

\section{RESULTADOS E DISCUSSÃO}

Não houve diferenças significativas quanto à ovoposição da traça entre os clones T13 e T14 e a testemunha com alto teor de AA T17 (Tabela 2). Estes clones obtiveram menores números de ovos (menor preferência para ovoposição) do que os demais tratamentos analisados. Embora não tão resistentes quanto T17 e os clones T13 e T14, os níveis de resistência dos clones com alto teor de AA (T1, T2, T4, T5, T9, T10, T11 e T12) foram maiores do que os apresentados pelas testemunhas $\mathrm{T} 15$, T16, T18 e por dois (T7 e T8) dos três clones BPX-370G com baixos teores de AA (Tabela 2).

Entre os clones com altos teores de AA, o T3 apresentou maior ovoposição pela traça, ficando este no mesmo grupo das testemunhas para baixo teor de AA (T15, T16, T18) e dos clones T7 e T8, com número de ovos até mesmo maiores do que os do clone T6 que apresenta baixo teor de AA. Tal resultado pode ser explicado pela presença de outros possíveis aleloquímicos com efeito antagônico idêntico ao da resistência causada por AA, bem como por diferenças entre os backgrounds genotípicos envolvidos, e mesmo por deficiências de amostragem no presente experimento. No entanto, em termos gerais fica evidente que altos teores de AA nos folíolos levaram a uma menor ovoposição da traça (contrastes C2, C3 da Tabela 2), embora a intensidade desta menor ovoposição possa ser influenciada pelo background genotípico considerado, uma vez que as ovoposições observadas nos clones BPX-410H foram em geral ligeiramente inferiores, em média, aos de clones BPX-370G também com altos teores de AA (Tabela 2).

A relação entre maiores teores de AA e menores níveis de ovoposição foi clara nos resultados do presente ensaio, onde os clones com altos teores de AA testados correspondem ao terceiro retrocruzamento para $S$. lycopersicum a partir do cruzamento interespecífico original S. lycopersicum x S. pennellii 'LA-716'. Estes resultados contrastam com os encontrados por Resende et al. (2006) que testaram populações mais próximas do material silvestre LA-716. Tais autores avaliaram populações $\mathrm{F}_{2}(S$. lycopersicum x S. pennellii ''LA-716) 
Tabela 1. Teores de AA (nmols/ $\mathrm{cm}^{2}$ área foliar) nos genótipos de tomateiro na geração $\mathrm{F}_{2} \mathrm{RC}_{3}$ e das testemunhas LA-716 e TOM-584 (acylsugars contents $\left(\mathrm{nmol} / \mathrm{cm}^{2}\right.$ leaflet área) genotypes $\mathrm{F}_{2} \mathrm{RC}_{3}$ cheks LA-716 and TOM-584). Lavras, UFLA, 2006.

\begin{tabular}{|c|c|c|}
\hline \multirow[b]{2}{*}{ Genótipos testados } & \multicolumn{2}{|c|}{ Nanomols de acilaçúcar $/ \mathrm{cm}^{2}$} \\
\hline & Média & $\begin{array}{l}\text { Amplitude } \\
\text { entre plantas }\end{array}$ \\
\hline $\begin{array}{l}\text { L.esculentum TOM-584 (50 plantas, em duas } \\
\text { observações repetidas }^{1} \text { ) }\end{array}$ & 0,225 & $0,172-0,361$ \\
\hline $\begin{array}{l}\text { L. pennellii LA-716 ( } 47 \text { plantas, em duas ob- } \\
\text { servações repetidas }^{1} \text { ) }\end{array}$ & 0,624 & $0,268-1,548$ \\
\hline $\begin{array}{l}\text { Populações } \quad F_{2} \text { (BPX-410H-01,-02,-03,-04) } \\
\left(400 \text { plantas, em duas observações repetidas }{ }^{1}\right)\end{array}$ & 0,277 & $0,106-1,019$ \\
\hline $\begin{array}{l}\text { Populações } \mathrm{F}_{2}(\mathrm{BPX}-410 \mathrm{H}-01,-02,-03,-04)(30 \\
\text { plantas selecionadas com altos teores de AA, } \\
\left.\text { em duas observações repetidas }^{1}\right)\end{array}$ & 0,530 & $0,409-1,019$ \\
\hline $\begin{array}{l}\text { Populações } \mathrm{F}_{2}(\mathrm{BPX}-410 \mathrm{H}-01,-02,-03,-04)(30 \\
\text { plantas selecionadas com altos teores de AA, } \\
\left.\text { em seis observações repetidas }{ }^{1}\right)\end{array}$ & 0,417 & $0,365-0,549$ \\
\hline $\begin{array}{l}\text { Populações } \mathrm{F}_{2}(\mathrm{BPX}-410 \mathrm{H}-01,-02,-03,-04)(15 \\
\text { plantas selecionadas com mais elevados teores } \\
\left.\text { de AA, em seis observações repetidas }{ }^{1}\right)\end{array}$ & $\begin{array}{c}0,470 \\
(75.4 \%)^{2}\end{array}$ & $\begin{array}{c}0,406-0,608 \\
(65.0 \%-97.4 \%)\end{array}$ \\
\hline
\end{tabular}

${ }^{1} \mathrm{O}$ número de observações refere-se aos testes de determinação dos teores de acilaçúcares (numbers of repeated observations refer to the tests of acylsugar content determination); ${ }^{2}$ Números entre parênteses representam percentagens relativas à média do acesso silvestre LA-716 (numbers within parentheses are percentages relative to the mean of the wild accession LA-716).

e $\mathrm{F}_{2} \mathrm{BC}_{1}[S$. lycopersicum 'TOM-584'x $\mathrm{F}_{2}$ (S. lycopersicum 'TOM-584' x $S$. pennellii 'LA716')] e não encontraram esta associação entre teores de AA e ovoposição do inseto. Por outro lado, essa associação entre menores ovoposições e maiores teores de AA também foram relatadas recentemente por Maluf et al. (2010), que, a exemplo do presente ensaio, também trabalhou com materiais provenientes de retrocruzamentos avançados. Isto indica uma associação entre maiores teores de AA e menores ovoposições da traça, embora esta associação possa ter sido mascarada nas primeiras gerações segregantes a partir do cruzamento interespecífico, provavelmente devido às grandes influências dos muitos "backgrounds" genotípicos existentes nestas gerações.

Foram detectadas diferenças significativas entre os tratamentos pelo teste Scott-Knott a 5\% de probabilidade para lesões nos folíolos (LF), porcentagem de folíolos atacados (PFA) e danos nas plantas (DP). Genótipos com altos teores de $\mathrm{AA}$ apresentaram maior resistência à traça-do-tomateiro quando comparados com os genótipos com baixos teores de AA, para as três variáveis consideradas (contrastes $\mathrm{C} 1, \mathrm{C} 2, \mathrm{C} 3$, Tabela 3).

Tanto para lesão nos folíolos (LF) quanto para percentagens de folíolos atacados (PFA) e danos na planta (DP), todos os clones com altos teores de AA demonstraram níveis substancialmente mais altos de resistência (menores níveis de dano) quando comparados com as testemunhas (T15, T16 e T18), pelo teste Scott-Knott de agrupamento de médias (Tabela 3). Estas diferenças também existiram quando as comparações dos clones de altos teores de AA foram feitas com os clones BPX-370G com baixos teores de AA, mas foram menos nítidas em alguns casos: os clones T2 e T3, com altos teores de $\mathrm{AA}$, não se apresentaram nitidamente distintos de alguns dos clones BPX-370G com baixos teores de AA com relação respectivamente, às variáveis PFA para o primeiro, e $L F$, PFA e DF para o segundo (Tabela 3). Estas pequenas discrepâncias relativas a comparações com os clones BPX-370G com baixos teores de AA não existiram quando os clones BPX-410H foram considerados, o que é indicativo, mais uma vez, da influência do background genotípico BPX-410H no sentido de potencializar os efeitos do alto teor de AA na resistência, relativamente ao efeito do background BPX-370G: os clones BPX-370G com altos teores de AA (T1, T2, T4 e T5), mesmo apresentando níveis de resistência mais elevados que os das testemunhas com baixo teor de AA, mostraram-se inferiores aos clones BPX-410H também com altos teores de AA (T9, T10, T13 e T14) (Tabela 3).

Dentre os clones com altos teores de AA, destacaram-se T9, T13 e T14, cujas médias foram significativamente menores que as dos demais clones testados. Estes clones foram apenas menos resistentes do que o T17 (S. pennellii), altamente resistente à $T$. absoluta. A maior resistência do $\mathrm{T} 17 \mathrm{em}$ relação à dos clones BPX-410H e BPX-370G selecionados para altos teores de AA pode ser atribuída tanto pela diferença nos teores de AA (os teores de AA no acesso silvestre são mais elevados), como por outros fatores de resistência, além dos AA, presentes em (S. pennellii), e não recuperados durante o processo de seleção, conforme sugerido por Maluf et al. (2010).

A associação entre níveis elevados de AA e a resistência à traça-do-tomateiro T. absoluta encontrada no presente ensaio com genótipos já com características agronômicas melhoradas, vêm sendo confirmada desde as gerações segregantes iniciais a partir do cruzamento interespecífico original S. lycopersicum x S. pennellii 'LA-716' (Resende et al., 2006; Pereira et al., 2008) até os retrocruzamentos mais avançados, com características agronômicas próximas das comerciais (Maluf et al. 2010).

Os dados deste trabalho reafirmam inferências de outros autores (Resende et al., 2003; Gonçalves et al., 2006; Resende et al., 2006; Saeidi et al., 2007; Maluf et al. 2010; Resende et al., 2008) de que os AA derivados de $S$. pennellii são o principal fator responsável pelos altos níveis de resistência a pragas e de que a seleção indireta para altos teores de AA é eficiente na obtenção de linhagens melhoradas de tomateiro com bons níveis de resistência. Por outro lado, AA podem não ser o único fator envolvido 
Tabela 2. Número de ovos $/ 2 \mathrm{~cm}^{2}$ de área foliar medido no terço superior de plantas de tomateiro aos 14 e 18 dias após infestação com Tuta absoluta Meyr. e estimativas de contrastes de interesse (egg counts per 2- $\mathrm{cm}^{2}$ leaflet measured on the upper third portion of tomato plants at 14 and 18 days after infestation with Tuta absoluta Meyr. and estimates of contrasts of interest). Lavras, UFLA, 2006.

\begin{tabular}{|c|c|}
\hline Tratamentos & Número de ovos \\
\hline $\mathrm{T} 1=\mathrm{BPX}-370 \mathrm{G}-30-380-68-08-02 \mathrm{pl} \# 192=$ clone com alto teor de AA & $4,000 \mathrm{~B}^{1}$ \\
\hline $\mathrm{T} 2=\mathrm{BPX}-370 \mathrm{G}-30-380-68-08-02 \mathrm{pl} \# 287=$ clone com alto teor de AA & $4,750 \mathrm{~B}$ \\
\hline $\mathrm{T} 3=\mathrm{BPX}-370 \mathrm{G}-30-380-68-08-04 \mathrm{pl} \# 269=$ clone com alto teor de AA & $8,750 \mathrm{C}$ \\
\hline $\mathrm{T} 4=\mathrm{BPX}-370 \mathrm{G}-30-380-68-08-05 \mathrm{pl} \# 270=$ clone com alto teor de AA & $3,750 \mathrm{~B}$ \\
\hline T5= BPX-370G-30-380-68-08-05pl\#283 = clone com alto teor de AA & $3,125 \mathrm{~B}$ \\
\hline $\mathrm{T} 6=\mathrm{BPX}-370 \mathrm{G}-30-380-68-08-02 \mathrm{pl} \# 045=$ clone com baixo teor de AA & $3,750 \mathrm{~B}$ \\
\hline $\mathrm{T} 7=\mathrm{BPX}-370 \mathrm{G}-30-380-68-08-04 \mathrm{pl} \# 036=$ clone com baixo teor de AA & $6,125 \mathrm{C}$ \\
\hline $\mathrm{T} 8=\mathrm{BPX}-370 \mathrm{G}-30-380-68-08-05 \mathrm{pl} \# 120=$ clone com baixo teor de AA & $7,250 \mathrm{C}$ \\
\hline $\mathrm{T} 9=\mathrm{BPX}-410 \mathrm{H}-01 \mathrm{pl} \# 281=$ clone com alto teor de AA & $4,750 \mathrm{~B}$ \\
\hline $\mathrm{T} 10=\mathrm{BPX}-410 \mathrm{H}-02 \mathrm{pl} \# 69=$ clone com alto teor de AA & $4,250 \mathrm{~B}$ \\
\hline $\mathrm{T} 11=\mathrm{BPX}-410 \mathrm{H}-03 \mathrm{pl} \# 345=$ clone com alto teor de AA & $3,500 \mathrm{~B}$ \\
\hline $\mathrm{T} 12=\mathrm{BPX}-410 \mathrm{H}-03 \mathrm{pl} \# 368=$ clone com alto teor de AA & $4,375 \mathrm{~B}$ \\
\hline $\mathrm{T} 13=\mathrm{BPX}-410 \mathrm{H}-04 \mathrm{pl} \# 348=$ clone com alto teor de AA & $1,250 \mathrm{~A}$ \\
\hline $\mathrm{T} 14=\mathrm{BPX}-410 \mathrm{H}-04 \mathrm{pl} \# 481=$ clone com alto teor de AA & $1,125 \mathrm{~A}$ \\
\hline $\mathrm{T} 15=\mathrm{TOM}-584=$ linhagem comercial com baixo teor de AA & $7,000 \mathrm{C}$ \\
\hline $\mathrm{T} 16=\mathrm{TOM}-598=$ linhagem comercial com baixo teor de AA & $8,500 \mathrm{C}$ \\
\hline $\mathrm{T} 17=\mathrm{LA}-716=$ acesso de Lycopersicon pennellii com alto teor de AA & $0,000 \mathrm{~A}$ \\
\hline $\mathrm{T} 18=\mathrm{BONUS}-\mathrm{F} 1=$ hibrido comercial com baixo teor de AA & $8,750 \mathrm{C}$ \\
\hline Identificação dos contrastes de interesse & Estimativa \\
\hline $\mathrm{C} 1=[$ clones BPX-370G com altos teores de AA] vs [clones BPX-370G com baixos teores] & $-0,8335 \mathrm{~ns}^{2}$ \\
\hline $\mathrm{C} 2=[$ clones BPX-370G $+\mathrm{BPX}-410 \mathrm{H}$ com altos teores de AA] vs [clones BPX-370G baixos teores] & $-1,7425^{*}$ \\
\hline $\begin{array}{l}\mathrm{C} 3=[\text { clones } \mathrm{BPX}-370 \mathrm{G}+\mathrm{BPX}-410 \mathrm{H} \text { com altos teores de } \mathrm{AA}] \text { vs. [clones BPX-370G+hibrido com- } \\
\text { ercial com baixos teores de AA] }\end{array}$ & $-4,1175^{* *}$ \\
\hline $\mathrm{C} 4=[$ clones com altos teores de AA $]$ vs. [L. pennellii LA-716] & $3,9659 * *$ \\
\hline
\end{tabular}

${ }^{1}$ médias seguidas da mesma letra, nas colunas, não diferem entre si pelo teste Scott Knott $(\mathrm{p}<0,05)($ means followed by the same letter within the column do not differ significantly from each other by Scott Knott test $(\mathrm{p}<0,05) ;{ }^{2} \mathrm{~ns}=$ não significativo a $5 \%$ de probabilidade pelo teste de $\mathrm{F}$ (ns= non-significant at $5 \%$ probability by the $\mathrm{F}$ test); $* *$, ${ }^{*}$ significativo a $1 \%$ e $5 \%$ de probabilidade respectivamente, pelo teste $\mathrm{F}$ $(* *, *$ significant at $1 \%$ and $5 \%$ probability respectively, by the $\mathrm{F}$ test).

na resistência à traça em $S$. pennellii, uma vez que o acesso silvestre possui níveis de resistência mais elevados do que os genótipos selecionados para altos teores de AA, uma conclusão semelhante à de Maluf et al. (2010). Contudo, além de serem o componente mais importante da resistência, os AA têm uma herança simples (Resende et al., 2002; Gonçalves et al., 2007), essencialmente monogênica, o que torna particularmente fácil sua introdução em linhagens-elite de tomateiro. Este fato, aliado à recente descoberta de Maluf et al. (2010) de que híbridos heterozigotos resultantes do cruzamento entre linhagens com altos teores de AA e linhagens com baixos teores de AA apresentaram níveis satisfatórios de resistência a artrópodos-praga (T. abso- luta, Bemisia argentifollii e Tetranychus urticae), trazem novas perspectivas para o melhoramento de tomateiro visando à resistência múltipla a pragas. Desta maneira, novos clones com altos teores de AA foram identificados, particularmente os clones BPX-410H-01pl\#281, BPX-410H-04pl\#348 e BPX-410H-04pl\#481 são promissores para o programa de melhoramento genético em tomateiro visando resistência à T. absoluta Meyr., e provavelmente a outras pragas, podendo ser utilizados tanto para obtenção de novas linhagens de tomateiro resistentes, quanto para a obtenção de novos híbridos (também resistentes) por meio de sua combinação com outras linhagens que possuam baixos teores de AA.

\section{AGRADECIMENTOS}

Os autores agradecem à FAPEMIG, CNPq/MCT, CAPES/MEC, UFLA, FAEPE (Fundação de Apoio ao Ensino, Pesquisa e Extensão), FUNDECC (Fundação de Desenvolvimento Científico e Cultural) e às empresas HortiAgro Sementes, Sakata Sudamerica e Sakata Seed Corporation (Japão) pelo auxílio financeiro, concessão de bolsas e disponibilização da infra-estrutura.

\section{REFERÊNCIAS}

FERREIRADF. 2000. Análises estatísticas por meio do Sisvar para Windows 4.0. In: REUNIÃO ANUAL DA REGIÃO BRASILEIRA DA SOCIEDADE INTERNACIONAL DE BIOMETRIA, 45. Anais... São Carlos, Universidade Federal de São Carlos. p. 255-258.

GONCALVES LD; MALUF WR; CARDOSO MG; GOMES LAA; NASCIMENTO IR. 
Tabela 3. Médias das notas para lesão nos folíolos (LF); porcentagem de folíolos atacados (PFA); danos na planta (DP) e estimativas dos contrastes de interesse entre genótipos de tomateiro, medidas 48 dias após infestação com Tuta absoluta Meyr. (score means for leaflet lesion types (LLT), percentage of leaflets attacked (PLA) and overall plant damage (OPD), and contrast estimative of interest among genotypes measured at 48 days after infestation with Tuta absoluta Meyr.). Lavras, UFLA, 2006.

\begin{tabular}{|c|c|c|c|}
\hline Tratamentos & $\mathbf{L F}$ & PFA & $\overline{\text { DP }}$ \\
\hline $\mathrm{T} 1=\mathrm{BPX}-370 \mathrm{G}-30-380-68-08-02 \mathrm{pl} \# 192=$ linhagem com alto teor de AA & $2,5313^{1} \mathrm{D}$ & $2,3750^{1} \mathrm{C}$ & $2,7813^{1} \mathrm{D}$ \\
\hline $\mathrm{T} 2=\mathrm{BPX}-370 \mathrm{G}-30-380-68-08-02 \mathrm{pl} \# 287=$ linhagem com alto teor de AA & $2,7813 \mathrm{D}$ & $2,8125 \mathrm{D}$ & $3,0313 \mathrm{D}$ \\
\hline $\mathrm{T} 3=\mathrm{BPX}-370 \mathrm{G}-30-380-68-08-04 \mathrm{pl} \# 269=$ linhagem com alto teor de AA & $3,0625 \mathrm{E}$ & $3,2188 \mathrm{D}$ & $3,3750 \mathrm{E}$ \\
\hline $\mathrm{T} 4=\mathrm{BPX}-370 \mathrm{G}-30-380-68-08-05 \mathrm{pl} \# 270=$ linhagem com alto teor de AA & $2,5625 \mathrm{D}$ & $2,5938 \mathrm{C}$ & $2,8750 \mathrm{D}$ \\
\hline $\mathrm{T} 5=\mathrm{BPX}-370 \mathrm{G}-30-380-68-08-05 \mathrm{pl} \# 283=$ linhagem com alto teor de AA & $2,7188 \mathrm{D}$ & $2,5938 \mathrm{C}$ & $2,9688 \mathrm{D}$ \\
\hline $\mathrm{T} 6=\mathrm{BPX}-370 \mathrm{G}-30-380-68-08-02 \mathrm{pl} \# 045=$ linhagem com baixo teor de AA & $3,2813 \mathrm{E}$ & $3,1563 \mathrm{D}$ & $3,5000 \mathrm{E}$ \\
\hline $\mathrm{T} 7=\mathrm{BPX}-370 \mathrm{G}-30-380-68-08-04 \mathrm{pl} \# 036=$ linhagem com baixo teor de AA & $3,1875 \mathrm{E}$ & $3,1250 \mathrm{D}$ & $3,3438 \mathrm{E}$ \\
\hline $\mathrm{T} 8=\mathrm{BPX}-370 \mathrm{G}-30-380-68-08-05 \mathrm{pl} \# 120=$ linhagem com baixo teor de AA & $3,4063 \mathrm{E}$ & $3,2188 \mathrm{D}$ & $3,6250 \mathrm{~F}$ \\
\hline $\mathrm{T} 9=\mathrm{BPX}-410 \mathrm{H}-01 \mathrm{pl} \# 281=$ linhagem com alto teor de AA & $2,0625 \mathrm{~B}$ & $1,8438 \mathrm{~B}$ & $2,0938 \mathrm{~B}$ \\
\hline $\mathrm{T} 10=\mathrm{BPX}-410 \mathrm{H}-02 \mathrm{pl} \# 69=$ linhagem com alto teor de AA & $2,2500 \mathrm{C}$ & $2,1250 \mathrm{C}$ & $2,4063 \mathrm{C}$ \\
\hline $\mathrm{T} 11=\mathrm{BPX}-410 \mathrm{H}-03 \mathrm{pl} \# 345=$ linhagem com alto teor de AA & $2,5938 \mathrm{D}$ & $2,5313 \mathrm{C}$ & $2,6250 \mathrm{D}$ \\
\hline $\mathrm{T} 12=\mathrm{BPX}-410 \mathrm{H}-03 \mathrm{pl} \# 368=$ linhagem com alto teor de AA & $2,3438 \mathrm{C}$ & $2,5000 \mathrm{C}$ & $2,7500 \mathrm{D}$ \\
\hline $\mathrm{T} 13=\mathrm{BPX}-410 \mathrm{H}-04 \mathrm{pl} \# 348=$ linhagem com alto teor de AA & $1,8750 \mathrm{~B}$ & $1,9375 \mathrm{~B}$ & $2,0625 \mathrm{~B}$ \\
\hline $\mathrm{T} 14=\mathrm{BPX}-410 \mathrm{H}-04 \mathrm{pl} \# 481=$ linhagem com alto teor de AA & $1,9688 \mathrm{~B}$ & $1,8438 \mathrm{~B}$ & $1,9375 \mathrm{~B}$ \\
\hline $\mathrm{T} 15=\mathrm{TOM}-584=$ linhagem comercial com baixo teor de AA & $3,6250 \mathrm{~F}$ & $3,5000 \mathrm{E}$ & $3,7813 \mathrm{~F}$ \\
\hline $\mathrm{T} 16=\mathrm{TOM}-598=$ linhagem comercial com baixo teor de AA & $4,0000 \mathrm{~F}$ & $3,7188 \mathrm{E}$ & $3,7813 \mathrm{~F}$ \\
\hline $\mathrm{T} 17=\mathrm{LA}-716=$ acesso de Lycopersicon pennellii alto teor de AA & $1,2500 \mathrm{~A}$ & $1,0000 \mathrm{~A}$ & $1,0000 \mathrm{~A}$ \\
\hline $\mathrm{T} 18=\mathrm{BONUS}-\mathrm{F} 1=$ hibrido comercial com baixo teor de AA & $3,7500 \mathrm{~F}$ & $3,5000 \mathrm{E}$ & $3,5938 \mathrm{~F}$ \\
\hline Identificação dos contrastes de interesse & \multicolumn{3}{|c|}{ Estimativa } \\
\hline $\begin{array}{l}\mathrm{C} 1=[\text { clones BPX-370G com altos teores de AA] vs [clones BPX-370G com } \\
\text { baixos teores] }\end{array}$ & $-0,5604 * *$ & $-0,4479 * *$ & $-0,4833 * *$ \\
\hline $\begin{array}{l}\mathrm{C} 2=[\text { clones BPX-370G }+\mathrm{BPX}-410 \mathrm{H} \text { com altos teores de AA] } v s \text { [clones BPX- } \\
370 \mathrm{G} \text { baixos teores }]\end{array}$ & $-0,8599 * *$ & $-0,7689 * *$ & $-0,8617 * *$ \\
\hline $\begin{array}{l}\mathrm{C} 3=[\text { clones } \mathrm{BPX}-370 \mathrm{G}+\mathrm{BPX}-410 \mathrm{H} \text { com altos teores de } \mathrm{AA}] \text { vs. [clones BPX- } \\
370 \mathrm{G}+\text { hibrido comercial com baixos teores de AA] }\end{array}$ & $-1,3599 * *$ & $-1,1752 * *$ & $-1,0909 * *$ \\
\hline $\mathrm{C} 4=[$ clones com altos teores de AA] vs. [L. pennellii LA-716] & $1,1818 * *$ & $1,3977 * *$ & $1,6279 * *$ \\
\hline
\end{tabular}

${ }^{1}$ médias seguidas da mesma letra, nas colunas, não diferem entre si pelo teste $\operatorname{Scott} \operatorname{Knott}(\mathrm{p}<0,05)$ (means followed by the same letter within the column do not differ from each other by Scott Knott test $(\mathrm{p}<0,05) ;{ }^{* *}$ significativo a $1 \%$ de probabilidade pelo teste $\mathrm{F}(* *$ significant at $1 \%$ probability by the $\mathrm{F}$ test).

2007. Herança de acilaçúcares em genótipos de tomateiro provenientes de cruzamento interespecífico. Pesquisa Agropecuária Brasileira 42: 699-705.

GONÇALVES LD; MALUF WR; CARDOSO MG; RESENDE JTV; CASTRO EM; SANTOS NM; NASCIMENTO IR; FARIA MV. 2006. Relação entre zingibereno, tricomas foliares e repelência de tomateiros a Tetranychus evansi. Pesquisa Agropecuária Brasileira 41: 267-273.

LABORY CRG; SANTA-CECILIA LVC; MALUF WR; CARDOSO MG; BEARZOTTI E; SOUZA JC. 1999. Seleção indireta para teor de 2-tridecanona em tomateiros segregantes e sua relação com a resistência à traça-dotomateiro. Pesquisa Agropecuária Brasileira 34: 733-740.

MALUF WR; BARBOSA LV; SANTACECÍLIA LC. 1997. 2-tridecanone-mediated mechanisms of resistance to the South american tomato pinworm Scrobipalpuloides absoluta (Meyrick, 1917) (LepidopteraGelechiidae) in Lycopersicon spp. Euphytica, 93: 189-194.

MALUF WR; MACIEL GM; GOMES LAA; CARDOSO MG; GONÇALVES LD; SILVA EC; KNAPP M. 2010. Broad-spectrum Arthropod Resistance in Hybrids between High- and Low-Acylsugar Tomato Lines. Crop Science 50: 439-450.

PEREIRA GVN; MALUF WR; GONÇALVES LD; NASCIMENTO IR; GOMES LAA; LICURSI V. 2008. Seleção para alto teor de acilaçúcares em genótipos de tomateiro e sua relação com a resistência ao ácaro vermelho (Tetranychus evansi) e à traça (Tuta absoluta). Ciência e Agrotecnologia 32: 996-1004.

RESENDE JTV; CARDOSO MG; MALUF WR; SANTOS CD; GONÇALVES LD; RESENDE LV; NAVES FO. 2002. Método colorimétrico para quantificação de acilaçúcar em genótipos de tomateiro. Ciência e Agrotecnologia 26: 1204-1208.
RESENDE JTV. 2003. Resistência a artrópodospragas, mediada poracilaçúcares em tomateiros obtidos do cruzamento interespecifico de Lycopersicon esculentum Mill 'TOM-584'x L. Pennellii 'LA-716'. Lavras: UFLA. 91p. (Tese doutorado).

RESENDE JTV; MALUF WR; FARIA MV; PFANN AZ; NASCIMENTO IR. 2006. Acylsugars in tomato leaflets confer resistance to the South American tomato pinworm, Tuta absoluta Meyr. Scientia Agrícola 63: 20-25.

RESENDE JTV; MALUF WR; CARDOSO MG; FARIA MV; GONCALVES LD; NASCIMENTO IR. 2008. Resistance of tomato genotypes with high level of acylsugars to Tetranychus evansi Baker \& Pritchard. Scientia Agricola 65:31-35.

SAEIDI Z; MALLIK B; KULKARNI RS. 2007. Inheritance of glandular trichomes and two-spotted spider mite resistance in cross Lycopersicon esculentum 'Nandi' and L. pennellii 'LA2963'. Euphytica 154: 231-238. 